\title{
2-dimensional models of rapidly rotating stars I. Uniformly rotating zero age main sequence stars
}

\author{
I. W. Roxburgh ${ }^{1,2}$ \\ 1 Astronomy Unit, Queen Mary, University of London, Mile End Road, London E1 4NS, UK \\ e-mail: I.W. Roxburgh@qmul .ac.uk \\ 2 LESIA, Observatoire de Paris, Place Jules Janssen, 92195 Meudon Cedex, France
}

Received 30 April 2004 / Accepted 6 August 2004

\begin{abstract}
We present results for 2-dimensional models of rapidly rotating main sequence stars for the case where the angular velocity $\Omega$ is constant throughout the star. The algorithm used solves for the structure on equipotential surfaces and iteratively updates the total potential, solving Poisson's equation by Legendre polynomial decomposition; the algorithm can readily be extended to include rotation constant on cylinders. We show that this only requires a small number of Legendre polynomials to accurately represent the solution. We present results for models of homogeneous zero age main sequence stars of mass $1,2,5,10 M_{\odot}$ with a range of angular velocities up to break up. The models have a composition $X=0.70, Z=0.02$ and were computed using the OPAL equation of state and OPAL/Alexander opacities, and a mixing length model of convection modified to include the effect of rotation. The models all show a decrease in luminosity $L$ and polar radius $R_{\mathrm{p}}$ with increasing angular velocity, the magnitude of the decrease varying with mass but of the order of a few percent for rapid rotation, and an increase in equatorial radius $R_{\mathrm{e}}$. Due to the contribution of the gravitational multipole moments the parameter $\Omega^{2} R_{\mathrm{e}}^{3} / G M$ can exceed unity in very rapidly rotating stars and $R_{\mathrm{e}} / R_{\mathrm{p}}$ can exceed 1.5 .
\end{abstract}

Key words. stars: interiors - stars: rotation

\section{Introduction}

Rapid rotation is one of the major problems inhibiting the use of stellar oscillations to diagnose the internal structure of stars and thereby to test and develop our understanding of stellar evolution. As a first step in seeking to address these problems we here report results on the construction of self consistent 2-dimensional models of rapidly rotating zero age main sequence stars, where the angular velocity is taken as constant throughout the star.

As is well known from the pioneering work of Von Zeipel (1924), Eddington (1929) and Sweet (1950), rotation drives circulation currents in the radiative zones of stars with velocities $v_{\text {circ }} \sim \alpha R / t_{\mathrm{KH}}$ where $t_{\mathrm{KH}}=R^{2} L / G M^{2}$ is the Kelvin Helmholtz thermal time scale and $\alpha=\Omega^{2} R^{3} / G M$ is the ratio of centrifugal force to gravity. These velocities are very small except possibly in the surface layers and at the boundaries between radiative and convective zones (neglecting overshooting). Although the circulation currents make a negligible contribution to hydrostatic balance they will transport angular momentum which, unless compensated by some other transport process, will change the initial angular velocity distribution.

In the absence of a magnetic field, the changing angular velocity field may become unstable and the resulting microturbulence transport angular momentum and provide some chemical mixing (cf. Roxburgh 1970; Zahn 1992). The time dependent evolution of $\Omega$ remains an unsolved problem; Mestel (1953) showed that except in very rapidly rotating stars the build up of chemical composition gradients can suppress the circulation; Zahn (1992) argued that horizontal mixing can take place along isentropic surfaces ensuring that $\Omega$ is constant on such surfaces, however if constant entropy and constant composition surfaces do not coincide, small horizontal variations in molecular weight may suppress such mixing. In very rapidly rotating stars the circulation may be fast enough so that the star approaches a steady state configuration where there are no circulation currents (cf. Roxburgh 1964); this problem will be addressed in a subsequent publication.

The situation is different if the star possesses a magnetic field. Even a very weak field can maintain (almost) uniform rotation and provide the torque needed to balance the advection of angular momentum by the circulation (cf. Roxburgh 1963). We here assume this to be the case and that the field is sufficiently weak so that its contribution to the hydrostatic support can be neglected. In what follows we shall assume that the star is uniformly rotating and in a quasi steady state with uniform composition. We defer to later work the consequences of relaxing these assumptions.

Since the circulation velocities are very much smaller than the rotation velocities they can be neglected in the hydrostatic balance, the steady state equations governing the structure in 
the radiative zones of a star rotating with constant angular velocity $\Omega$ can then be expressed as (cf. Roxburgh et al. 1965)

$$
\begin{aligned}
& \frac{\nabla P}{\rho}=-\nabla \Phi+\Omega^{2} w=-\nabla \Psi \\
& \boldsymbol{F}_{\mathrm{r}}=-\frac{16 \sigma T^{3}}{3 \kappa \rho} \nabla T \\
& \rho \boldsymbol{v} \cdot \nabla U-\frac{P}{\rho} \boldsymbol{v} \cdot \nabla \rho=\epsilon \rho-\nabla \cdot \boldsymbol{F}_{\mathrm{r}} \\
& \nabla^{2} \Phi=4 \pi G \rho
\end{aligned}
$$

where

$\Psi=\Phi-\frac{1}{2} \Omega^{2} w^{2}, \quad \nabla^{2} \Psi=4 \pi G \rho-2 \Omega^{2}$

$P, \rho, T, \Phi$ are the pressure, density temperature and gravitational potential, $\Psi$ is the total potential defined by Eq. (5), $\boldsymbol{F}_{\mathrm{r}}$ the radiative flux, $U, \kappa, \epsilon$ the internal energy, opacity and energy generation per unit mass, $\boldsymbol{v}$ the velocity of meridional circulation and $w=r \sin \theta$ the distance from the rotation axis. It follows from Eq. (1) that $P, \rho$ are constant on equipotential surfaces $\Psi=$ const., or $P=P(\Psi), \rho=\rho(\Psi)$. The equation of state gives $P=P\left(\rho, T, X_{i}\right)$ so if the composition $X_{i}$ is constant on equipotential surfaces then $T=T(\psi)$. Since we are here only considering homogeneous stars this condition is automatically satisfied and hence $\kappa=\kappa(\Psi), \epsilon=\epsilon(\Psi)$.

As is well known (cf. Roxburgh et al. 1965) in a steady state the net advection of energy by the circulation across an equipotential surface $\Psi=$ const. is zero, so on integrating the energy Eq. (4) over a volume $V_{\psi}$ within such a surface and using Gauss's theorem gives

$\int_{S_{\psi}} \boldsymbol{F}_{\mathrm{r}} \cdot \mathrm{d} \boldsymbol{S}=-\frac{16 \sigma T^{3}}{3 \kappa \rho} \frac{\mathrm{d} T}{\mathrm{~d} \Psi} \int_{S_{\psi}} \nabla \Psi \cdot \mathrm{d} \boldsymbol{S}=\int_{V_{\psi}} \epsilon \rho \mathrm{d} V=L_{\Psi}$

where $L_{\Psi}$ is the total energy produced within the volume $V_{\psi}$ and is therefore a function of $\Psi$. On using Eq. (5) and Gauss's theorem we have

$\int_{S_{\psi}} \nabla \Psi . \mathrm{d} S=\int_{V_{\psi}} \nabla^{2} \Psi \mathrm{d} V=4 \pi G M_{\psi} C(\Psi)$

where

$C(\Psi)=1-\frac{\Omega^{2} V_{\psi}}{2 \pi G M_{\psi}}, \quad$ and $\quad M_{\psi}=\int_{V_{\psi}} \rho \mathrm{d} V$

is the mass within an equipotential surface $\Psi=$ const.

The effect of rotation on convection in astrophysical conditions is poorly understood. In the case of the Sun surface observations and helioseismic inversions show that the angular velocity in the convective envelope varies with both angle and depth. One possible explanation could be that in a rotating star the turbulent convection is anisotropic and inhomogeneous resulting in a latitude variation in the convective flux, circulation currents and differential rotation. The differential rotation could well drive dynamo generation of magnetic fields in both convective cores and convective envelopes. which again would also effect the energy transport. Efforts to model this behaviour have yet to be developed to a stage where we can be confident that we understand the underlying physics, to proceed we shall make some simplifying assumptions and defer to later work a more sophisticated treatment of the interaction of rotation and convection. We ignore differential rotation taking $\Omega=$ const. so that $P, \rho, T$ are constant on surfaces $\Psi=$ const., ignore possible convective overshooting and assume that convection sets in when

$\nabla=\frac{P}{T} \frac{\mathrm{d} T}{\mathrm{~d} P}=\nabla_{\mathrm{ad}}$

where $\nabla=\nabla(\Psi)$. The convective flux $\boldsymbol{F}_{\mathrm{c}}$ is given by a local mixing length model in which eddies rise and fall along the direction of effective gravity (cf. Appendix A) which gives

$\boldsymbol{F}_{\mathrm{c}}=F_{\mathrm{c}}\left(P, T, \rho, \nabla, \nabla_{\mathrm{ad}}\right) \boldsymbol{n}$

where $\boldsymbol{n}$ is the unit normal to a surface $\Psi=$ const. The magnitude $F_{\mathrm{c}}$ is therefore constant on equipotential surfaces, $F_{\mathrm{c}}=$ $F_{\mathrm{c}}(\Psi)$. We incorporate the convective flux into Eq. (6) to give

$-\frac{64 \pi G \sigma M T^{3}}{3 \kappa \rho} \frac{\mathrm{d} T}{\mathrm{~d} \Psi} C(\Psi)+F_{\mathrm{c}}(\Psi) S_{\psi}=L_{\Psi}$

where $S_{\psi}$ is the surface area of an equipotential surface $\Psi=$ const.

We now introduce the radial variable $s$ as the distance from the centre of the star to an equipotential $\Psi=\Psi_{\mathrm{s}}$ along an (arbitrarily) prescribed angle $\theta_{f}$, and define factors $W, A, B, C, D$ as functions of $M_{\mathrm{s}}$, or equivalently of $s$, such that

$V_{\psi}=\frac{4 \pi s^{3}}{3} W, \quad S_{\psi}=4 \pi s^{2} A, \frac{\mathrm{d} V_{\psi}}{\mathrm{d} s}=4 \pi s^{2} D$

$\frac{\mathrm{d} \Psi}{\mathrm{d} s}=\frac{G M_{\mathrm{s}}}{s^{2}} B, \quad C=1-\frac{2}{3} \frac{\Omega^{2} s^{3}}{G M_{\mathrm{s}}} W$

and the structure equations can then be expressed as

$\frac{\mathrm{d} P}{\mathrm{~d} s}=-\frac{G M_{\mathrm{s}}}{s^{2}} B$

$\frac{\mathrm{d} M_{\mathrm{s}}}{\mathrm{d} s}=4 \pi \rho s^{2} D$

$\frac{\mathrm{d} L_{\mathrm{s}}}{\mathrm{d} s}=4 \pi \epsilon s^{2} D$

$-\frac{64 \pi \sigma T^{3} s^{2}}{3 \kappa \rho} \frac{\mathrm{d} T}{\mathrm{~d} s} \frac{C}{B}+4 \pi s^{2} F_{\mathrm{c}} A=L_{\mathrm{s}}$.

If the factors $A, B, C, D$ were known as functions of $s, M_{\mathrm{s}}$, these are ordinary differential equations for $s, P, \rho, T, L$ as a function of $M_{\mathrm{s}}$ and could be solved by simply incorporating these factors into a standard stellar structure/evolution code. The factors are not known and have to be calculated self consistently from the total potential $\Psi$ given by the solution of the partial differential Poisson Eq. (5). This is done iteratively as described in the following section.

In previous studies by Faulkner et al. (1968), Kippenhahn \& Thomas (1970) and Papaloizou \& Whelan (1973), the factors $A, B, C, D$ were not determined self consistently but from some approximate estimate. In Faulkner et al. (1968), the reference angle $\theta_{f}=\cos ^{-1}(1 / \sqrt{3})$, and $A, B, C, D$ were approximated by $A=D=1$ and

$B=1-\alpha, \frac{B}{C}=1+50.8 \alpha^{5.27}, \quad \alpha=\frac{2}{3} \frac{\Omega^{2} s^{3}}{G M_{\mathrm{s}}}$ 
the approximation for $B$ coming from 1st order perturbation analysis and that for $C$ from the Roche envelope models of Roxburgh et al. (1965). In the analysis of Kippenhahn \& Thomas (1970) and Papaloizou \& Whelan (1973) all the factors were computed from a simple Roche model; the latter authors discussed a possible iterative scheme similar to the one used here but did not implement it.

The method adopted here is closely related to the self consistent field method of Jackson (1970) where the potential was evaluated from the integral representation of the solution of Poisson's equation; here we use spectral decomposition.

\section{Method of solution}

The structure Eqs. (14)-(17) are solved on a fixed mesh $M(i), i=0, N_{i}$, and the shape of the equipotential surfaces specified by a set of scale factors $x_{k}(i)$ at a set of specified angles $\theta_{k}, k=0, N_{k}$, such that the points $\left(r_{k}(i), \theta_{k}\right)$ lie on the equipotential through $\left(s(i), \theta_{f}\right)$ where $r_{k}(i)=s x_{k}(i)$. The iteration scheme is as follows:

1. With estimates of $A(i), B(i), C(i), D(i)$ we solve the structure equations to determine $s(i), \rho(i)$.

2. Since $\rho$ is constant on equipotential surfaces the estimate of the $x_{k}(i)$ gives the density $\rho(r, \theta)$ on the 2-dimensional array $r_{k}(i), \theta_{k}$.

3. Solving Poisson's equation with this distribution of $\rho$ gives the gravitational potential $\Phi(r, \theta)$ and hence the total potential $\Psi(r, \theta)$.

4. With this value of $\Psi(r, \theta)$ we determine new values of $A(i), B(i), C(i), D(i)$, new values of the $r_{k}(i)$ for the points on the equipotentials through $s(i), \theta_{f}$ and hence new values of the scale factors $x_{k}(i)=r_{k}(i) / s(i)$.

This scheme is iterated to convergence when the values of $A(i), B(i), C(i), D(i)$ remain the same to $1: 10^{6}$.

The iteration can be started from the spherically symmetric values $A(i)=B(i)=C(i)=D(i)=x_{k}(i)=1$, or from a known solution with different $\Omega$, or from the approximation scheme of Faulkner et al. (1968).

\subsection{Solution of the Poisson equation for $\Phi(r, \theta)$}

After stage 2 above we have $\rho$ on the mesh $r_{k}(i), \theta_{k}$. We then interpolate $\rho$ on to the polar coordinate mesh $\left(s(i), \theta_{k}\right)$. This distribution of $\rho$ and the corresponding gravitational potential $\Phi$ are then expressed as finite sums of Legendre polynomials

$$
\begin{gathered}
\rho(s, \theta)=\sum_{0}^{N_{k}} c_{k}(s) P_{2 k}(\cos \theta) \\
\Phi(s, \theta)=\sum_{0}^{N_{k}} f_{k}(s) P_{2 k}(\cos \theta)
\end{gathered}
$$

where the $f_{k}$ satisfy the equations

$\frac{1}{s^{2}} \frac{\mathrm{d}}{\mathrm{d} s}\left(s^{2} \frac{\mathrm{d} f_{k}}{\mathrm{~d} s}\right)-\frac{2 k(2 k+1)}{s^{2}} f_{k}=4 \pi G c_{k}$ subject to the boundary conditions $f_{k}(0)=0, k \neq 0$ and

$(2 k+1) f_{k}+s \frac{\mathrm{d} f_{k}}{\mathrm{~d} s}=0$ at $s=R_{0}$

where $R_{0}$ is any spherical surface outside the star.

The decomposition of the density was done algebraically by simply demanding that (at each radius $s(i)$ ) Eq. (19) be satisfied at the angles $\theta_{k}$, and solving the resulting matrix equations to give

$c_{k}=W_{k n}^{-1} \rho_{n}, \quad W_{k n}=P_{2 k}\left(\cos \theta_{n}\right), k, n=0, N_{k}$.

This simple algorithm worked remarkably well, much better than seeking to determine the $c_{k}$ by integrating $\rho(s, \theta) P_{2 k}(\cos \theta)$ over sphere using a much finer mesh in angle.

Having determined the $c_{k}$ the coefficients $f_{k}$ were determined from an integral representation of the solution of Eqs. (21)-(22) namely

$f_{k}=s^{2 k} \int_{R_{0}}^{s} \frac{4 \pi G}{3 s^{4 k+2}}\left[\int_{0}^{s} c_{k}(s) s^{2 k+2} \mathrm{~d} s\right] \mathrm{d} s-\lambda_{k} s^{2 k}$

where

$\lambda_{k}=\frac{4 \pi G}{(4 k+1) R_{0}^{4 k+1}} \int_{0}^{R_{0}} c_{k}(s) s^{2 k+2} \mathrm{~d} s$.

Provided one sets $c_{k}=0$ when it is sufficiently small (e.g. $\left.\left|c_{k}\right|<10^{-10} \rho_{\mathrm{c}}\right)$ this simple algorithm works well. Detailed tests of this and the density decomposition algorithm are reported in Sect. 4 below, where we show that they successfully reproduce the analytic results for models of inhomogeneous spheroids where the density is constant over spheroidal surfaces but varies with polar radius.

\subsection{Calculation of the factors $A, B, C, D, x_{k}$}

With the values of $f_{k}(s)$ determined, the value of $\Psi(s, \theta)$ can be calculated on a fine mesh in angle $\left(s, \theta_{j}\right), j=0, N_{j}$ using Eq. (20) for $\Phi$ and the definition of the potential $\Psi$

$\Psi\left(s, \theta_{j}\right)=\Phi\left(s, \theta_{j}\right)-\frac{\Omega^{2} s^{2} \sin ^{2} \theta_{j}}{2}$.

The values of $r_{j}(i)$ at angles $\theta_{j}$ which have the same value of $\Psi$ as the values at the reference points $\left(s(i), \theta_{f}\right)$ are then obtained by interpolation on the mesh $\left(s, \theta_{j}\right)$. It is convenient to take the mesh $\theta_{j}$ to be such that the mesh $\theta_{k}$ is a subset of the mesh $\theta_{j}$.

With these $\left(r_{j}, \theta_{j}\right)$ the area $S_{\psi}$ and volume $V_{\psi}$ of equipotential surfaces can be calculated by quadrature, and the values of $\mathrm{d} \Psi / \mathrm{d} s, \mathrm{~d} V_{\psi} / \mathrm{d} s$ determined by numerical differentiation. Values of the factors $A(i), B(i), C(i), D(i)$ are then determined from their definitions in Eqs. (12) and (13). The interpolations were done using a cubic routine and the integrations by Simpson's rule. This process can be repeated until the values of $A, B, C, D$ converge within this step. In fact this is not necessary but the whole iterative scheme converges faster by taking about 3 iterations in this step.

The values of shape factors $x_{k}(i)=r_{k}(i) / s(i)$ at the angles $\theta_{k}$ are then calculated and stored to give to give the first approximation to the $r_{k}(i)$ in the next iteration. 


\subsection{Solution of the structure equations}

The structure Eqs. (14)-(17) were solved using a scaled down version of my stellar evolution code, setting time derivatives to zero, using a fixed composition $(X=0.70, Z=0.02)$ and equilibrium $p p$ and $C N$ cycle nuclear reactions, and incorporating the factors $A, B, C, D$. The equation of state was OPAL EOS2001 (Rogers \& Nayfonov 2001) and the opacities a smooth blend of OPAL GN93 (Iglesias \& Rogers 1996) and Alexander \& Ferguson (1994) tables; interpolation in the tables was by local splines which ensures continuity of first derivatives. Convection was described by the local mixing length model (see Appendix A) with $\alpha=1.8$, and convective mixing is treated as a diffusion process with the diffusion coefficient determined by the convective model. The structure equations are discretised to 2 nd order on the mesh in $M(i)$ and solved by relaxation, the solution is taken as having converged when the relative changes in all variables, $\delta V_{i} / V_{i}$, are less than some specified value, normally set as $1 / N_{i}^{2}$ where $N_{i}$ is the number of mesh points.

\subsection{Surface boundary conditions}

The structure of the surface layers of rotating stars is another area where our understanding is poor and effort needs to be put in to understand the physics of these layers. This is the case even for a slowly rotating star like the Sun where a better understanding of the structure of outer layers is needed before we can determine the expected shape of the solar surface. This is a problem with a long history going back to the interpretations of the solar oblateness measured by Dicke \& Goldenberg (1967). Osaki (1966) proposed that the distribution of angular velocity is such that there is no meridional circulation and that this distribution differs little from that of uniform rotation. We shall here retain the assumption of uniform rotation in the surface layers, deferring to subsequent work a more detailed study of the atmosphere.

Since $P, T$ are then constant on equipotential surfaces the surface boundary condition must also be constant on equipotentials. My evolution code has a simple grey Eddington atmosphere which is incorporated into the model by the simple expedient of imposing the surface boundary condition $T^{4}=$ $0.75 T_{\text {eff }}^{4}(\tau+2 / 3), P=g \tau / \kappa$, high up in the radiative atmosphere an optical depth $\tau \approx 0.01-0.001$ Since in a rotating star the flux $\boldsymbol{F}$, and hence the local $T_{\text {eff }}$, and the local value of effective gravity $|\nabla \Psi|$, vary over an equipotential surface, we replace them by $T_{\mathrm{s}}, g_{\mathrm{s}}$, their values averaged over the surface equipotential

$T_{\mathrm{s}}^{4}=\frac{L}{\sigma S_{\psi}}=\frac{L}{4 \pi \sigma s^{2} A}, \quad g_{\mathrm{s}}=\frac{\int \nabla \Psi \cdot \mathrm{d} S}{S_{\psi}}=\frac{G M}{s^{2}} \frac{C}{A}$

where $S_{\psi}$ is the area of the equipotential, and take the boundary condition as $T^{4}=0.75 T_{\mathrm{s}}^{4}(\tau+2 / 3), P=g_{\mathrm{s}} \tau / \kappa$ at some $\tau$. This condition is then independent of the angle $\theta_{f}$ along which we calculate the model.

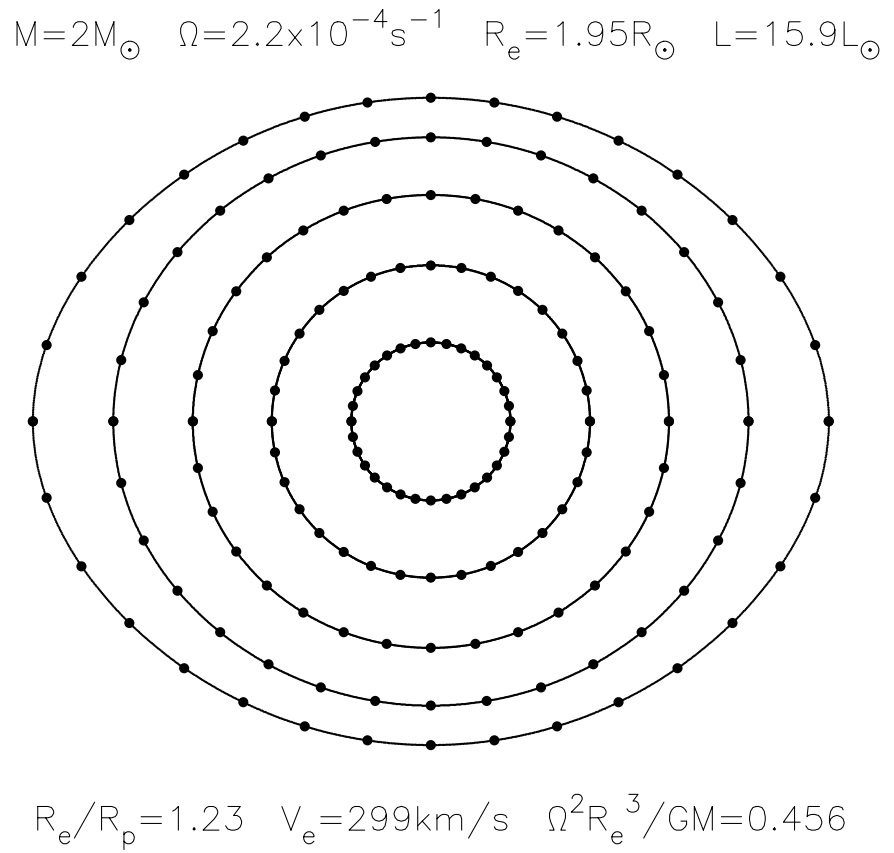

Fig. 1. Equipotential surfaces in a model of $2 M_{\odot}$ with an angular velocity $\Omega=2.2 \times 10^{-4} \mathrm{rad} / \mathrm{s}$. The points on these equipotentials are the fitting points $\left(r_{k}, \theta_{k}\right)$.

\subsection{Mesh resolution and accuracy}

Most models were computed taking the reference angle $\theta_{f}=$ $\pi / 2$, ie along the equator; with $N_{i}=1000$ for the mesh in $M_{i}$, $N_{k}=8$ for the solution of Poisson's equation, and $N_{j}=360$ for the computation of the factors $A, B, C, D$. All of $\theta_{f}, N_{i}, N_{j}, N_{k}$ were varied to check that the accuracy of the calculations was of the order of $1: 10^{5}$. Details are given in Sect. 4 below. The advantage of taking $\theta_{f}=\pi / 2$ is that it was not necessary to extend the radial mesh beyond the surface when calculating the gravitational potential.

\section{Results}

Figure 1 shows the equipotential surfaces in a zams star of $2 M_{\odot}$ rotating with angular velocity $\Omega=2.2 \times 10^{-4} \mathrm{rad} / \mathrm{s}$ and equatorial velocity of $299 \mathrm{~km} \mathrm{~s}^{-1}$. The model was computed with the angular variation of $\rho, \Phi$ modelled by Legendre polynomials $P_{2 k}, k=0,8$, fitted at angles $\theta_{k}=k \pi / 2, k=0,8$. The reference angle along which the model was computed was $\theta_{f}=\pi / 2$, the radial mesh was $N_{i}=1000$ and the angular mesh $N_{j}=360$. Figure 2 shows the variation of the factors $A, B, C, D$ with radius for this model

Tables 1-4 list the properties of a family of models with masses $1,2,5,10 M_{\odot}$ for a range of angular velocities. In all cases the luminosity and polar radius decreases with increasing angular velocity. as was found to be the case in earlier work using a two zone perturbation model (Faulkner et al. 1968).

Note that for very rapid rotation the ratio of equatorial to polar radius $R_{\mathrm{e}} / R_{\mathrm{p}}$ can exceed 1.5 and the parameter $\alpha=$ $\Omega^{2} R_{\mathrm{e}}^{3} / G M$ can exceed unity. This is primarily due to the contribution of the gravitational quadrupole moment, $\Phi_{1}(r) P_{2 k}$ which enhances the gravitational attraction in the equatorial 


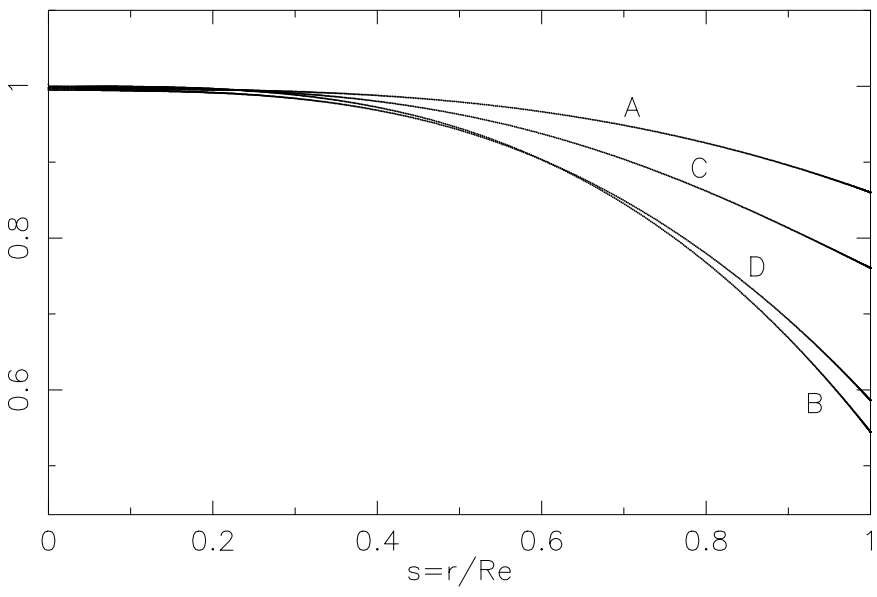

Fig. 2. Variation with radius of the geometrical factors $A, B, C, D$ with equatorial radius for the model in Fig. 1.

Table 1. Models of a uniformly rotating star of $1 M_{\odot}$.

\begin{tabular}{lcccccc}
\hline \hline $10^{4} \Omega$ & 0 & 1.0 & 3.0 & 4.0 & 4.6 & 4.6254 \\
\hline$\alpha$ & 0.000 & 0.020 & 0.205 & 0.451 & 0.903 & 1.0018 \\
$R_{\mathrm{e}} / R_{\mathrm{p}}$ & 1.000 & 1.010 & 1.108 & 1.237 & 1.470 & 1.5198 \\
$V_{\mathrm{e}} k / s$ & 0 & 64 & 201 & 288 & 381 & 395 \\
$L / L_{\odot}$ & 0.712 & 0.705 & 0.650 & 0.599 & 0.561 & 0.5595 \\
$R_{\mathrm{e}} / R_{\odot}$ & 0.914 & 0.919 & 0.964 & 1.035 & 1.189 & 1.2261 \\
$R_{\mathrm{p}} / R_{\odot}$ & 0.914 & 0.909 & 0.871 & 0.837 & 0.809 & 0.8067 \\
\hline
\end{tabular}

Table 2. Models of a uniformly rotating star of $2 M_{\odot}$.

\begin{tabular}{lcccccc}
\hline \hline 1.0 & 2.0 & 2.2 & 2.4 & 2.4168 & & \\
\hline$\alpha$ & 0.000 & 0.056 & 0.318 & 0.456 & 0.815 & 1.0003 \\
$R_{\mathrm{e}} / R_{\mathrm{p}}$ & 1.000 & 1.028 & 1.160 & 1.230 & 1.409 & 1.5020 \\
$V_{\mathrm{e}} k / s$ & 0 & 114 & 257 & 299 & 373 & 400 \\
$L / L_{\odot}$ & 16.59 & 16.46 & 16.06 & 15.94 & 15.81 & 15.800 \\
$R_{\mathrm{e}} / R_{\odot}$ & 1.603 & 1.644 & 1.843 & 1.951 & 2.233 & 2.3800 \\
$R_{\mathrm{p}} / R_{\odot}$ & 1.603 & 1.599 & 1.589 & 1.587 & 1.585 & 1.5846 \\
\hline
\end{tabular}

regions. This behaviour was found in investigations on the structure of rotating polytropes by perturbation analysis (Monaghan \& Roxburgh 1965) and in two dimensional spectral models of rapidly rotating polytropes (Roxburgh unpublished). This effect is enhanced if the rotation increases inwards or increases towards the rotation axis for rotation that is constant on cylinders.

\section{Testing the accuracy of the solutions}

Several factors limit the numerical accuracy of the solutions: the accuracy of the stellar structure code which depends on the radial mesh $N_{i}$; the order $N_{k}$ of the Legendre representation of the angular variation; the angular mesh $N_{j}$ on which the functions $A, B, C, D$ are evaluated; and the accuracy of the algorithms for solving Poisson's equation.

The dependence on the radial mesh can be simply tested by varying $N_{i}$ when solving a non rotating model; the results of
Table 3. Models of a uniformly rotating star of $5 M_{\odot}$.

\begin{tabular}{lcccccc}
\hline \hline $10^{4} \Omega$ & 0 & 0.05 & 1.0 & 1.7 & 1.84 & 1.8485 \\
\hline$\alpha$ & 0.000 & 0.024 & 0.105 & 0.482 & 0.848 & 1.0007 \\
$R_{\mathrm{e}} / R_{\mathrm{p}}$ & 1.000 & 1.012 & 1.053 & 1.243 & 1.427 & 1.5036 \\
$V_{\mathrm{e}} k / s$ & 0 & 92 & 191 & 379 & 469 & 497 \\
$L / L_{\odot}$ & 541 & 538 & 531 & 513 & 508 & 507 \\
$R_{\mathrm{e}} / R_{\odot}$ & 2.623 & 2.650 & 2.744 & 3.202 & 3.667 & 3.8629 \\
$R_{\mathrm{p}} / R_{\odot}$ & 2.623 & 2.618 & 2.505 & 2.575 & 2.569 & 2.5691 \\
\hline
\end{tabular}

Table 4. Models of a uniformly rotating star of $10 M_{\odot}$.

\begin{tabular}{lcccccc}
\hline \hline $10^{4} \Omega$ & 0 & 0.05 & 1.0 & 1.3 & 1.45 & 1.4601 \\
\hline$\alpha$ & 0.000 & 0.039 & 0.188 & 0.421 & 0.817 & 1.0011 \\
$R_{\mathrm{e}} / R_{\mathrm{p}}$ & 1.000 & 1.028 & 1.160 & 1.229 & 1.407 & 1.5053 \\
$V_{\mathrm{e}} k / s$ & 0 & 137 & 292 & 417 & 540 & 579 \\
$L / L_{\odot}$ & 5711 & 5664 & 5523 & 5392 & 5313 & 5307 \\
$R_{\mathrm{e}} / R_{\odot}$ & 3.880 & 3.943 & 4.195 & 4.610 & 5.347 & 5.6965 \\
$R_{\mathrm{p}} / R_{\odot}$ & 3.880 & 3.866 & 3.829 & 3.799 & 3.785 & 3.7844 \\
\hline
\end{tabular}

Table 5. Accuracy of solutions of the structure equations.

\begin{tabular}{ccccc}
\hline \hline$N_{i}$ & $\delta R / R$ & $\delta L / L$ & $\delta \rho_{\mathrm{c}} / \rho_{\mathrm{c}}$ & $\delta T_{\mathrm{c}} / T_{\mathrm{c}}$ \\
\hline 4000 & $1.8 \times 10^{-6}$ & $7.8 \times 10^{-7}$ & $8.0 \times 10^{-7}$ & $4.7 \times 10^{-7}$ \\
2000 & $8.1 \times 10^{-6}$ & $3.7 \times 10^{-6}$ & $3.8 \times 10^{-6}$ & $1.4 \times 10^{-6}$ \\
1000 & $3.3 \times 10^{-5}$ & $1.6 \times 10^{-5}$ & $1.7 \times 10^{-5}$ & $6.5 \times 10^{-6}$ \\
\hline
\end{tabular}

such a test for a $2 M_{\odot}$ star are given in Table 5 where we give the fractional change of key variables with mesh size relative to values with a mesh of $N_{i}=8000$. In the results for rotating star presented here we use a radial mesh with $N_{i}=1000$ so there is no point in solving the angular structure to any greater accuracy than $3 \times 10^{-5}$.

The error in the representation of the density $\rho$ by finite series of Legendre Polynomials is indicated in Fig. 3 which shows the maximum variation in $\rho / \rho_{\mathrm{c}}$ over equipotential surfaces calculated on the fine mesh $r_{j}(i), \theta_{j}, j=0,360, i=$ 0,1000 and using a Legendre series with $N_{k}=8$, for the reference $2 M_{\odot}$ star given in Fig. 1 . The error in $\rho / \rho_{\mathrm{c}}<5 \times 10^{-9}$ and the corresponding error in $\Phi$ is even less due to the long range nature of gravity and the cancellation due to errors of opposite signs. The major inaccuracies are due to the inaccuracy of the finite Legendre representation of the density on spheres which cross the boundary of the convective core, and those which cross the surface.

To test the accuracy as a function of $N_{k}$ we compare solutions with $N_{i}=1000, N_{j}=360$ and different values of $N_{k}$, with the solution with the highest resolution $N_{j}=720, N_{k}=24$ for the particular case $M=2 M_{\odot}$. The reference angle $\theta_{f}=\pi / 2$, that is along the equator, to ensure comparability in the radial mesh. In Table 6 we give the error in the equatorial radius $\delta R_{\mathrm{e}} / R_{\mathrm{e}}$ for values of $\Omega=0.5,1,1.5,2,2.2,2.4 \times 10^{-4} \mathrm{~s}^{-1}$ and for $N_{k}=1,2,4,8,12$. The solutions with $N_{k} \geq 4$ give accuracies better than $10^{-5}$ over the whole range of $\Omega$ and even 


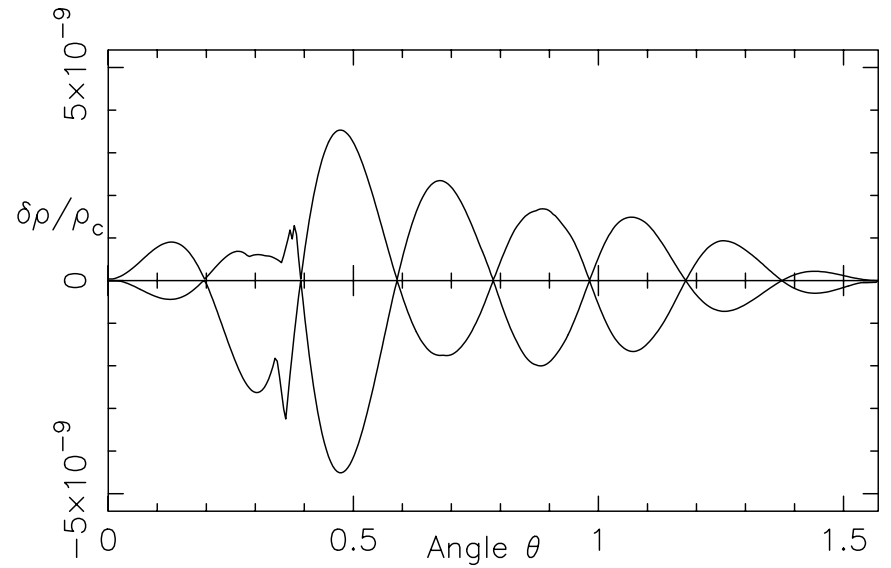

Fig. 3. Maximum errors in fitting the density over equipotential surfaces using a Legendre series with $N_{k}=8$ for the model in Fig. 1.

Table 6. Accuracy of solutions as a function of $N_{k} \Omega$ in units of $10^{-4} \mathrm{~s}^{-1}$.

\begin{tabular}{lcccccc}
\hline \hline$\Omega$ & 0.5 & 1.0 & 1.5 & 2.0 & 2.2 & 2.4 \\
\hline$N_{k}$ & & & & & & \\
12 & $10^{-9}$ & $10^{-9}$ & $10^{-9}$ & $10^{-9}$ & $10^{-9}$ & $4 \times 10^{-8}$ \\
8 & $10^{-9}$ & $10^{-9}$ & $10^{-9}$ & $10^{-9}$ & $10^{-9}$ & $4 \times 10^{-8}$ \\
4 & $10^{-9}$ & $10^{-9}$ & $10^{-9}$ & $10^{-9}$ & $8 \times 10^{-9}$ & $2 \times 10^{-7}$ \\
2 & $10^{-9}$ & $1 \times 10^{-8}$ & $2 \times 10^{-7}$ & $1 \times 10^{-6}$ & $2 \times 10^{-6}$ & $1 \times 10^{-5}$ \\
1 & $6 \times 10^{-8}$ & $2 \times 10^{-6}$ & $2 \times 10^{-5}$ & $1 \times 10^{-4}$ & $3 \times 10^{-4}$ & $2 \times 10^{-3}$ \\
\hline
\end{tabular}

for large values of $\Omega$ the solutions are adequately represented by $N_{k}=2$. The solutions with $N_{k}=1$ correspond to the first order perturbation analysis as presented by Faulkner et al. (1968) and adequately represent the solutions for moderate $\Omega$.

To test the simple algorithms for the spectral decomposition and solution of Poisson's equation, described by Eqs. (23)-(25), we derived analytic solutions for the gravitational potential inside an inhomogeneous spheroid with variable density (see Appendix B), and compared this with the solution obtained using the numerical algorithms. By an inhomogeneous spheroid we mean a body whose surface is defined by

$\frac{w^{2}}{R_{\mathrm{e}}^{2}}+\frac{z^{2}}{R_{\mathrm{p}}^{2}}=1$

where $w=r \sin \theta, z=r \cos \theta, R_{\mathrm{p}}^{2}=R_{\mathrm{e}}^{2}\left(1-e^{2}\right)$ with $e$ the eccentricity, and where the density is constant on similar spheroidal surfaces of eccentricity $e$ but which varies from spheroid to spheroid, the variation being prescribed by $\rho(z)$, the density along the minor axis.

The gravitational potential at a point $\left(w_{i}, z_{i}\right)$ inside such a body can be determined analytically as (see Appendix B)

$$
\begin{aligned}
V= & \frac{4 \pi G}{e \sqrt{1-e^{2}}}\left[\tan ^{-1}\left(\frac{e}{\sqrt{1-e^{2}}}\right) \int_{z_{1}}^{R_{\mathrm{p}}} \rho(z) z \mathrm{~d} z\right. \\
& \left.+\int_{0}^{z_{1}} \tan ^{-1}\left(\frac{e z}{\sqrt{\left(z^{2}+\lambda\right)\left(1-e^{2}\right)}}\right) \rho(z) z \mathrm{~d} z\right]
\end{aligned}
$$

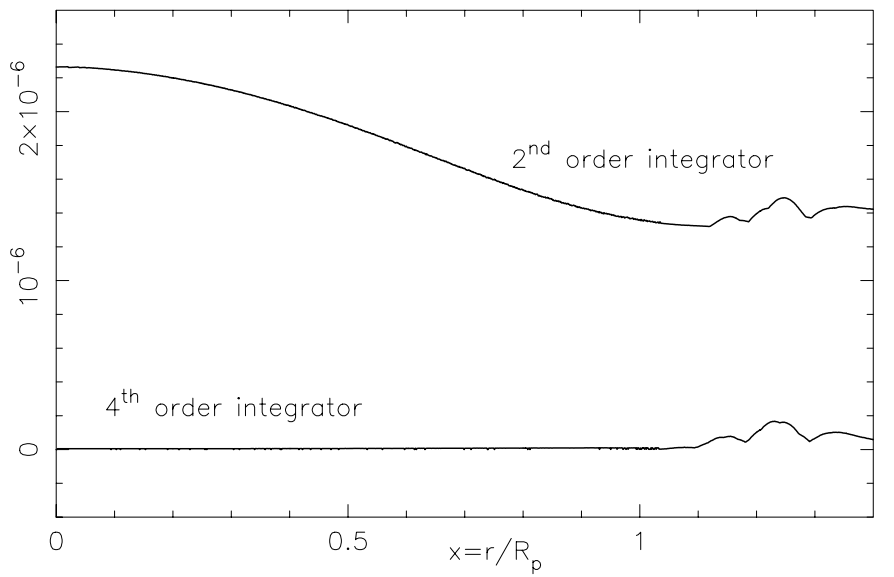

Fig. 4. Error in using the Poisson solver algorithm with $N_{k}=8$ for the potential of an inhomogeneous spheroid with $R_{\mathrm{e}} / R_{\mathrm{p}}=1.4$ for both 2 nd and 4 th order integrators.

where $\lambda(z)$ is the largest root of

$\frac{w_{i}^{2}\left(1-e^{2}\right)}{z_{i}^{2}+\lambda\left(1-e^{2}\right)}+\frac{z_{i}^{2}}{z^{2}+\lambda}=1$

and $z_{1}$, the value of $z$ on the polar axis of the spheroid through $\left(w_{i}, z_{i}\right)$, is given by

$z_{1}^{2}=w_{i}^{2}\left(1-e^{2}\right)+z_{i}^{2}$.

The potential outside the body $\left(z_{1}>R_{\mathrm{p}}\right)$ is given solely by the second term in Eq. (29).

To test the solution algorithms we took a model with $R_{\mathrm{e}} / R_{\mathrm{p}}=1.4$ with the density distribution given by

$\rho(z)=\rho_{\mathrm{c}}\left(1-\frac{z^{2}}{R_{\mathrm{p}}^{2}}\right)^{4}$

and evaluated the potential on a mesh of $N_{i}=1000$ in $z$ and $N_{j}=64$ in $\theta$ using the analytical results above, and then solved the same model using the numerical spectral solution algorithms (Eqs. (19)-(25)) with $N_{k}=8$.

Figure 4 shows the difference between the two solutions for two cases: one where the integration in the Poisson solver (Eq. (25)) was a simple 2nd order trapezoidal rule, the second using a 4th order Simpson's rule. Even for the trapezoidal integrator the error, though systematic, is well within the accuracy limit of $10^{-5}$. The 4 th order integrator gives much better accuracy and the figure shows that the main source of error is in modelling the outer layers where a spherical surface intersects the surface of the "star".

Since the density is constant on spheroidal surfaces with the same eccentricity, the spheroidal model is considerably more distorted in the inner regions than is a uniformly rotating star, where the eccentricity of equipotential surfaces is given approximately by $e^{2}=\Omega^{2} r^{3} / G M_{\mathrm{r}}$ which is small in the central regions.

We conclude that the solutions presented are accurate to order of $3 \times 10^{-5}$, and could readily be improved by using a finer mesh in $N_{i}, N_{j}$. 

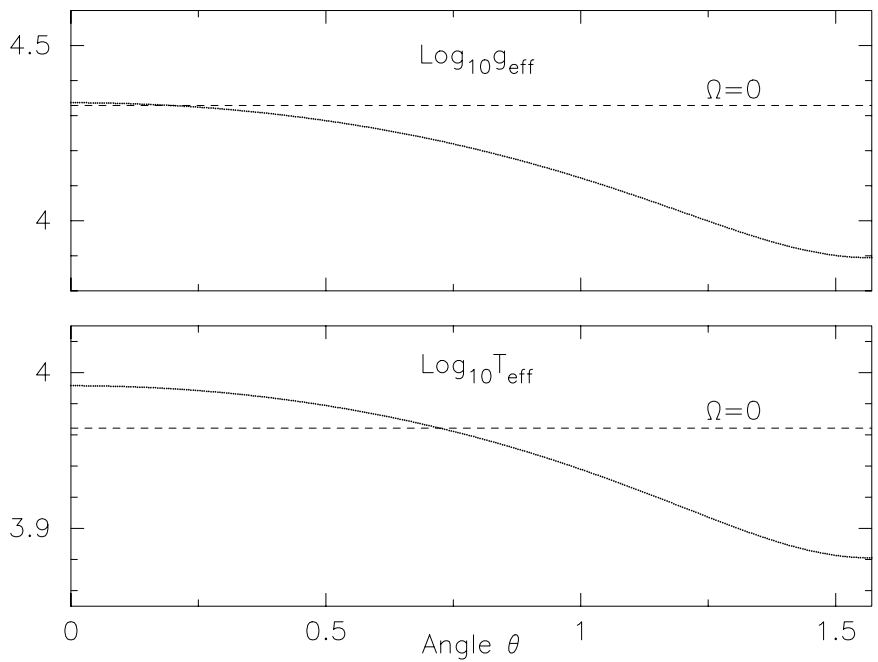

Fig. 5. Variation of effective gravity $g_{\mathrm{e}}$ and von Zeipel effective temperature $T_{\text {eff }}$ over the surface of the model in Fig. 1.

\section{Observational consequences}

In several studies of the effect of rotation on stellar structure efforts have been made to calculate the effect of rotation and orientation on the observational parameters e.g. $M_{v}, B-V$ and line profiles (cf. Sweet \& Roy 1953; Collins 1963, 2004). These calculations determine the variation over the stellar surface of effective temperature using the von Zeipel result $T_{\text {eff }}^{4} \propto$ $g_{\mathrm{e}}$ where $g_{\mathrm{e}}=|\nabla \Psi|$ is the effective gravity, and then build plane parallel atmospheres at each point on the surface with these $g_{\mathrm{e}}, T_{\text {eff }}$, integrating over the visible surface to obtain the values of $M_{v}, B-V$ and spectral line profiles. For illustrative purposes we show in Fig. 5 the variation of $g_{\mathrm{e}}$ and $T_{\text {eff }}$ computed in this way for the reference $2 M_{\odot}$ model in Fig. 1 .

Such calculations should be treated with caution since, as pointed out in Sect. 2.4 above, our knowledge of the detailed structure and dynamics of the surface layers of rapidly rotating stars is poor, and urgently needs to be developed in some depth. There may well be latitudinal motions and differential rotation which invalidate the simple application of the von Zeipel result.

\section{Conclusion}

We have constructed 2-dimensional models of homogeneous rotating stars where the rotation has been taken to be constant throughout the whole of the star. The method used is to solve the equations averaged over equipotential surfaces together with a spectral solution of Poisson's equation for the gravitational potential. The method gives accurate models, as accurate in the angular variation as in the radial variation of the spherical models which are limited by both the number of mesh points and the accuracy of interpolation in the equation of state and opacity tables. Results for models of 1, 2, 5, $10 M_{\odot}$ and a range of angular velocities are given in Tables 1 to 4 .

The method is readily adapted to differential rotation of the form $\Omega=\Omega(w)$, where $w=r \sin \theta$, by replacing the rotational potential $\Omega^{2} w^{2} / 2$ by $\int \Omega^{2}(w) w \mathrm{~d} w$

The results presented here suffer from many simplifications.
We have taken $\Omega=$ const. throughout the star, in both radiative layers and in convective cores and envelopes. The example of the Sun suggests this is a poor approximation at least for convective envelopes.

The possible effect of rotation on the onset of convective instability has been ignored and the onset taken as the point where $\nabla=\nabla_{\mathrm{ad}}$. Convective energy transport has been calculated using the local mixing length model with variable gravity averaged over an equipotential surface. This is a very crude model and detailed investigations are required to develop a more realistic model of convection which captures the true nature of the interaction of rotation and convection (and probably differential rotation).

The surface layers have been assumed to rotate uniformly and a simple grey atmosphere boundary condition derived on this basis. Much more effort needs to be put into understanding the structure and dynamics of the atmospheres of rotating stars, this is especially important when one seeks to determine the observable properties of rotating stars where one needs to have realistic 2-dimensional models of their atmospheres.

\section{Appendix A: Treatment of convection}

As stated in Sect. 1 since our understanding of the interaction of rotation and convection is poor we here make the simplifying assumption that the angular velocity is constant in convective regions. We make a simple modification of the mixing length model in which the bubbles are assumed to rise (fall) along the direction of effective gravity $\boldsymbol{g}_{\mathrm{e}}=\nabla \Psi$, due to their density deficiency (excess) and to be in pressure equilibrium with their surroundings so

$v \frac{\mathrm{d} v}{\mathrm{~d} n}=\frac{1}{2} g \frac{\delta T}{T} \chi, \quad$ where $\chi=-\left(\frac{\partial \log \rho}{\partial \log T}\right)_{P}$.

The factor $1 / 2$ is assumed to allow for viscous drag.

The temperature excess is taken as due to the rise (fall) of the bubble along the superadiabatic temperature gradient subject to radiative losses and is given by

$v \frac{\mathrm{d} \delta T}{\mathrm{~d} n}=v\left[\left(\frac{\mathrm{d} T}{\mathrm{~d} n}\right)_{\mathrm{ad}}-\frac{\mathrm{d} T}{\mathrm{~d} n}\right]-\left(\frac{48 \sigma T^{3}}{c_{\mathrm{p}} \kappa \rho^{2} \ell^{2}}\right) \delta T$

where $\ell=\alpha H_{\mathrm{p}}$ is the mixing length, $H_{\mathrm{p}}=P / g_{\mathrm{e}} \rho$ is the pressure scale height, $\alpha$ an adjustable parameter and the factor 48 depends on the assumed shape of the bubbles and is here taken to agree with the model of Bohm-Vitense (1958).

Defining $u=-\log P$, these equations reduce to

$v \frac{\mathrm{d} v}{\mathrm{~d} u}=\mathcal{A} \delta T, \quad \frac{\mathrm{d} \delta T}{\mathrm{~d} u}=C-\mathcal{B} \frac{\delta T}{v}$

where

$\mathcal{A}=\frac{1}{2} \chi \frac{P}{\rho T}, \quad \mathcal{B}=\frac{48 \sigma T^{3} g_{\mathrm{e}}}{c_{\mathrm{p}} \kappa \alpha^{2} P \rho}, \quad C=T \Delta \nabla$

$\Delta \nabla=\nabla-\nabla_{\mathrm{ad}}, \quad \nabla=\frac{\mathrm{d} \log T}{\mathrm{~d} \log P}, \quad \nabla_{\mathrm{ad}}=\left(\frac{\mathrm{d} \log T}{\mathrm{~d} \log P}\right)_{\mathrm{ad}}$. 
Variations of $\mathcal{A}, \mathcal{B}, \mathcal{C}$ over $\ell$ are neglected in this model so Eq. (A.3) have the solution $v=v_{0} u, \delta T=\delta T_{0} u$. Since bubbles are assumed to have travelled on average a distance $\ell / 2$, the change in $u,[u]=\alpha / 2$ and

$v=v_{0} \frac{\alpha}{2}, \quad \delta T=v_{0}^{2} \frac{\alpha}{2 \mathcal{A}} \quad$ with $v_{0}^{2}+\mathcal{B} v_{0}-\mathcal{A C}=0$.

The convective flux is in the direction of gravity and is estimated as

$\boldsymbol{F}_{\mathrm{c}}=\left\langle c_{\mathrm{p}} \rho v \delta T\right\rangle \boldsymbol{n}=f \frac{c_{\mathrm{p}} \rho \alpha^{2} v_{0}^{3}}{4 \mathcal{A}} \boldsymbol{n}$

where $f$ accounts for the averaging over upward and downward moving bubbles. Away from the centre $v$ and $\delta T$ are assumed to be perfectly correlated so $f=1$.

In the central regions $H_{\mathrm{p}}=P / g \rho \rightarrow \infty$ so we replace $H_{\mathrm{p}}$ by $H=\left(r H_{\mathrm{p}}\right)^{1 / 2}$ and set $f=H / H_{\mathrm{p}}$ which gives $F_{\mathrm{c}} \propto r$ as $r \rightarrow 0$.

The energy equation integrated over an equipotential surface is

$\int_{\Psi}\left(\boldsymbol{F}_{\mathrm{c}}+\boldsymbol{F}_{\mathrm{r}}\right) \cdot \mathrm{d} \boldsymbol{S}=L_{\psi}$

which in terms of $v_{0}$ becomes

$\frac{c_{\mathrm{p}} \rho \alpha^{2}}{4 \mathcal{A}} \int_{\psi} v_{0}^{3} \mathrm{~d} S+\left(\frac{16 \sigma T^{4}}{3 \kappa P} \nabla\right) \int_{\psi} \nabla \Psi . \mathrm{d} S=L \Psi$.

With $v_{0}$ given by Eq. (A.6) in principle this gives $\nabla$ in terms of $P, \rho, T, L$.

When radiative losses are unimportant $v_{0}$ and hence $F_{\mathrm{c}}$ are constant on equipotential surfaces, to retain this behaviour when radiative losses are important we replace $g_{\mathrm{e}}$ in $\mathcal{B}$ by its average over an equipotential surface $g_{\mathrm{s}} . g_{\mathrm{s}}$ and $\nabla_{\text {rad }}$ are defined by

$g_{\mathrm{s}}=\frac{1}{S_{\psi}} \int_{\psi} \nabla \Psi . \mathrm{d} S, \quad L_{\psi}=\frac{16 \sigma T^{4} g_{\mathrm{s}}}{3 \kappa P} \nabla_{\mathrm{rad}} S_{\psi}$

and on substituting into Eq. (A.9) we obtain

$\left(\frac{3 \kappa P c_{\mathrm{p}} \rho \alpha^{2}}{64 \sigma \mathcal{A} T^{4} g_{\mathrm{s}}}\right) v_{0}^{3}+\left(\frac{v_{0}^{2}+B_{0} v_{0}}{A T}\right)=\left(\nabla_{\mathrm{rad}}-\nabla_{\mathrm{ad}}\right)$

which is identical in form to the non rotating case except that $g_{\mathrm{s}}$ replaces $g$.

The solution for $v_{0}$ is obtained by setting $v_{0}=x \mathcal{B}$ so that $x$ satisfies the cubic

$x^{3}+\frac{4}{9}\left(x^{2}+x\right)=U=\frac{2}{9} \chi\left(\frac{P}{\rho \mathcal{B}^{2}}\right)\left(\nabla_{\mathrm{rad}}-\nabla_{\mathrm{ad}}\right)$.

Since $U>0$ there is only one real solution for $x$ which can most conveniently be found by the very rapidly convergent iterative scheme

$x_{0}=\frac{9 U}{(8+27 U)^{2 / 3}}, \quad x_{j+1}=\frac{U+2 x_{j}^{3}+4 x_{j}^{2} / 9}{3 x_{j}^{2}+8 x_{j} / 9+4 / 9}$.

With $x=v_{0} / \mathcal{B}$ determined Eq. (A.6) give

$\Delta \nabla=\mathcal{B}^{2}\left(\frac{x^{2}+x}{\mathcal{A} T}\right), \quad \nabla=\nabla_{\mathrm{ad}}+\Delta \nabla$.
In terms of the geometrical factors in the solution scheme introduced in Sect. 2 we have

$\nabla_{\mathrm{rad}}=\frac{3 \kappa P L_{\mathrm{s}}}{64 \pi \sigma G M_{\mathrm{s}} T^{4}} \frac{1}{C(s)}, \quad g_{\mathrm{s}}=\frac{G M_{\mathrm{s}}}{s^{2}} \frac{C(s)}{A(s)}$.

Such factors are readily incorporated into the mixing length subroutine in the stellar structure code.

\section{Appendix B: The gravitational potential of inhomogeneous spheroids}

The gravitational potential of uniform density spheroids and ellipsoids was studied in depth in the 18th and 19th centuries and much of this work is described in MacMillan (1930) and Ramsey (1940). These results can be used to determine the gravitational potential of spheroidal distributions of matter where the density is constant on similar spheroidal surfaces, but varies from surface to surface.

Consider a shell of uniform density $\rho$ bounded by two similar concentric spheroids, that is the spheroids with the same eccentricity,

$\frac{x^{2}}{a^{2}}+\frac{y^{2}}{a^{2}}+\frac{z^{2}}{c^{2}}=1$

$\frac{x^{2}}{a^{2}}+\frac{y^{2}}{a^{2}}+\frac{z^{2}}{c^{2}}=(1-\mathrm{d} k)^{2}$.

The mass of the shell is $\mathrm{d} M=4 \pi \rho a^{2} c \mathrm{~d} k$; the gravitational potential of the shell at a point $P=\left(x_{i}, y_{i}, z_{i}\right)$ is

$V=-\frac{1}{2} G \mathrm{~d} M \int_{\lambda}^{\infty} \frac{\mathrm{d} u}{\left(a^{2}+u\right) \sqrt{c^{2}+u}}$

where $\lambda=0$ for a point interior to the shell, whereas for a point exterior to the shell $\lambda$ is the largest root of the cubic

$\frac{x_{i}^{2}}{a^{2}+\lambda}+\frac{y_{i}^{2}}{a^{2}+\lambda}+\frac{z_{i}^{2}}{c^{2}+\lambda}=1$

(cf. Ramsey 1940). The integral $I_{\lambda}$ in Eq. (B.3) can be evaluated in closed form as

$I_{\lambda}=2 \frac{\sqrt{1-e^{2}}}{e c} \tan ^{-1}\left(\frac{e c}{\sqrt{\left(c^{2}+\lambda\right)\left(1-e^{2}\right)}}\right)$

where $e=\sqrt{a^{2}-c^{2}} / a$ is the eccentricity of the spheroid.

Now consider a distribution of matter such that the density $\rho$ is constant on similar spheroidal surfaces but varies from surface to surface. Let the outer surface of the spheroid be

$\frac{x^{2}}{a^{2}}+\frac{y^{2}}{a^{2}}+\frac{z^{2}}{c^{2}}=1$

and define the density distribution by its value $\rho(z)$ up the minor axis of the spheroid. An elemental spheroidal shell between $(z, z+\mathrm{d} z)$ has mass of $\mathrm{d} M=4 \pi \rho z^{2} \mathrm{~d} z /\left(1-e^{2}\right)$. At a point $P=\left(x_{i}, y_{i}, z_{i}\right)$ the contribution to the potential from spheroidal shells exterior to $P$ is

$V_{\mathrm{ext}}=\frac{4 \pi G}{e \sqrt{1-e^{2}}} \tan ^{-1}\left(\frac{e}{\sqrt{1-e^{2}}}\right) \int_{z_{i}}^{c} \rho(z) z \mathrm{~d} z$ 
and the contribution from shells interior to $z_{i}$ is

$V_{\text {in }}=\frac{4 \pi G}{e \sqrt{1-e^{2}}} \int_{0}^{z_{i}} \tan ^{-1}\left(\frac{e z}{\sqrt{\left(z^{2}+\lambda\right)\left(1-e^{2}\right)}}\right) \rho(z) z \mathrm{~d} z$

where $\lambda(z)$ is the largest root of

$\frac{x_{i}^{2}+y_{i}^{2}}{a_{z}^{2}+\lambda}+\frac{z_{i}^{2}}{z^{2}+\lambda}=1, \quad a_{z}^{2}=\frac{z^{2}}{1-e^{2}}$.

Given $\rho(z)$ the potential at any point, interior or exterior to the spheroid, is now readily evaluated by quadrature.

\section{References}

Alexander, D. R., \& Ferguson, J. W. 1994, ApJ, 437, 891

Bohm Vitense, E. 1958, Zs. f. Ast., 46, 108

Collins, G. W. II 1963, ApJ, 138, 1134

Collins, G. W. II 2004, in Stellar Rotation, ed. A. Maeder, \& P. Eenens, IAU Symp., 215, in press

Dicke, R. H., \& Goldenberg, H. M. 1967, Phys. Rev. Lett., 18, 313

Eddington, A. S. 1929, MNRAS, 90, 54
Faulkner, J., Roxburgh, I. W., \& Strittmatter, P. A. 1968, ApJ, 151, 203

Jackson, S. 1970, ApJ, 161, 579

Kippenhahn, R., \& Thomas, H. C. 1970, In Stellar Rotation, ed. A. Sletteback (Gordon \& Breach), IAU Coll., 4, 20

MacMillan, W. 1930, Theory of the Potential (Dover Edition, 1958)

Mestel, L. 1953, MNRAS, 113, 716

Monaghan, J., \& Roxburgh, I. W. 1965, MNRAS, 129, 5

Osaki, Y. 1966, Pub. Ast. Soc. Japan, 18, 70

Iglesias, C. A., \& Rogers, F. J. 1996, ApJ, 464, 943

Papaloizou, J. C. B., \& Whelan, J. A. J. 1973, MNRAS, 164, 1

Rogers, F. J., \& Nayfonov, A. 2002, ApJ, 576, 1064

Ramsey, H. 1940, Newtonian Attraction (Cambridge Univ. Press)

Roxburgh, I. W. 1963, MNRAS, 126, 67

Roxburgh, I. W. 1964, MNRAS, 128, 157, ibid, 237

Roxburgh, I. W. 1970, In Stellar Rotation, ed. A. Sletteback (Gordon \& Breach), IAU Coll., 4, 9

Roxburgh, I. W., Griffiths, S., \& Sweet, P. 1965, Zs. f. Ast., 61, 221

Sweet, P. A. 1950, MNRAS, 110, 548

Sweet, P. A., \& Roy, A. 1953, MNRAS, 113, 701

von Zeipel, H. 1924, MNRAS, 84, 665

Zahn, J.-P. 1992, A\&A, 265, 115 